\title{
NOTAS INTRODUTÓRIAS SOBRE A RELAÇÃO ENTRE A TEORIA DO CAPITAL SOCIAL E ATIVIDADE FÍSICA
}

\author{
Eduard Angelo Bendrath \\ Universidade Estadual de Maringá, Ivaiporã, Paraná, Brasil
}

\begin{abstract}
Resumo
O objetivo da presente revisão foi tentar estabelecer possíveis associações entre a teoria do capital social e atividade física a partir de estudos internacionais publicados na base de dados ScienceDirect. Baseado na abordagem qualitativa, e usando do processo de revisão sistemática de literatura, buscaram-se artigos com a temática proposta através de descritores e operadores lógicos. Analisou-se o capital social a partir da estrutura e intensidade que fundamenta as relações sociais de uma comunidade a partir da confiança, solidariedade, reciprocidade e participação cívica. Sugere-se que a atividade física e estado de saúde podem ser considerados fatores resultantes do acúmulo de capital social, potencializando a criação e manutenção de círculos virtuosos de participação popular reduzindo os índices de inatividade física.
\end{abstract}

Palavras-chave: Capital Social; Atividade Física; Saúde; Comunidade.

\section{Introdução}

O objetivo desse trabalho é traçar um panorama acerca das aproximações teóricas em relação ao conceito de capital social e a atividade física como mecanismo facilitador do desenvolvimento individual e coletivo. Nesse sentido, entende-se o capital social como um conceito relativo à intensidade das múltiplas relações sociais agregadas a um sistema de confiança mútuo, garantindo cooperação e tratamento igual entre indivíduos ou grupos, de acordo com os interesses e objetivos específicos em comum dentro de uma sociedade.

A partir dessa perspectiva, a atividade física compreendida a partir de vertentes diretas para a profilaxia e mudanças de comportamento social poderia atuar de forma a configurar uma rede de interações benéficas, não apenas para o indivíduo, mas também para todo o seu entorno. A atividade física pode ser desenvolvida e ampliada a partir de ações cotidianas em situações variadas da vida coletiva, sendo fomentadas a partir da criação e manutenção de redes de relacionamentos sociais que tendem a se solidificar com o tempo.

Para a construção do panorama de análise foram realizadas buscas na base de dados ScienceDirect ${ }^{1}$ a partir dos descritores "social capital", "physical activity" e "health", com foco específico na relação entre o capital social e atividade física. A definição teórica das bases do capital social foi conduzida a partir dos pressupostos defendidos por Portes (2000), Fukuyama (2002) e Putnam (2006). A análise tomou como princípio o entendimento de que o ambiente, a percepção social e a participação ativa na comunidade cívica são fatores que carregam em si sentidos que reforçam a coesão e eficácia coletiva, tais mecanismos sendo essenciais para a transformação da sociedade. Esses princípios fortalecem o estabelecimento

\footnotetext{
${ }^{1}$ Base de dados aberta da Elsevier que possui mais de 2.500 periódicos cadastrados e concentra $1 / 4$ de todo o conteúdo científico revisado por pares disponível no mundo. Para mais informações:< http://www.sciencedirect.com/>.
} 
de normas sociais fundamentalmente aceitas por todos, que de maneira objetiva, carregam valores que produzem resultados concretos para a vida coletiva.

Compreender, portanto, como o engajamento social e a adesão e participação frente à prática regular de atividade física são desenvolvidas, ampliando a confiança social das comunidades e fortalecendo os mecanismos de valoração do capital social local, tornou-se o foco desse texto. Dessa forma, objetivou-se levantar posições norteadoras de caráter introdutório (haja vista a escassez de trabalhos com a temática no Brasil), sobre a teoria do capital social e a possível relação com a atividade física em pesquisas consolidadas ao redor do mundo.

\section{Sobre o conceito de Capital Social}

O que chama a atenção para o uso atual do termo capital social faz menção ao fato de que o desenvolvimento socioeconômico, que inclui dentre outros fatores o bem estar físico e mental, não está apenas e unicamente relacionado a formas monetárias de capital, e sim a sua variabilidade de fatores que, definitivamente, englobam recursos provenientes da vida social. Essa variabilidade induz que ações nas mais diversas esferas, derivadas de objetivos comuns, favorecem seu acúmulo e proporcionam a instituição de princípios, valores, normas e condutas aceitas em determinado espaço/tempo.

Para Bourdieu (1985, p.248) apud Portes (2000, p. 134), o termo capital social é verificado como a junção de recursos efetivos ou potenciais ligados à posse de uma rede durável de relações mais ou menos institucionalizadas de conhecimento ou reconhecimento mútuo. Para Bourdieu (1985) todas as demais formas de capital seriam uma forma de conversibilidade e redução em última instância ao capital econômico monetário. Já de acordo com os conceitos de Loury $(1977 ; 1981)$ apud Portes $(2000)$, a forma como o qual o termo capital vem sendo empregado remete a um conceito demasiadamente individualista, centrando-se em um campo de competição nivelado e focado em competências. Os estudos de Loury fazem menção às formas desiguais de acesso às diferentes formas de oportunidade. Segundo Portes (2000), os trabalhos de Loury serviram de base para a construção das idéias de Coleman (1988) sobre a aproximação direta entre capital humano e capital social.

No entanto, Putnam (2006) remete o conceito de capital social a fatores ligados às estruturas sociais de cooperação, solidariedade, confiança e tolerância; para o autor, a comunidade cívica possui papel fundamental na definição do estado democrático das sociedades. Os conceitos de Putnam (2006) sobre a influência do capital social sobre os processos de democracia em diferentes contextos consideram as múltiplas relações estabelecidas entre a política e sociedade e a forma como tais relações são responsáveis pelo processo de desenvolvimento. Assim, a vida em um mundo marcado pelo sistema econômico capitalista parece ser impossível de estar atrelada a um conceito de puro altruísmo e vida coletiva em detrimento ao estímulo dos interesses próprios.

Putnam (2006) reforça o argumento em favor do capital social recorrendo a Tocqueville, enfatizando que a comunidade cívica e os cidadãos devem buscar o "interesse próprio corretamente estendido", ou seja, dentro dos contextos das necessidades públicas gerais. Considera-se pertinente a posição de Midgley (2007) que contrapõe tais definições destacando que o conceito de capital social ainda está mal definido, porém vem sendo usado atualmente em análises da sociedade civil para designar o volume e a intensidade das relações sociais cooperativas nas comunidades. Esse volume e intensidade nas relações sociais estão, portanto, como as estruturas fundamentais no processo de análise, pois, segundo Putnam (2006), o Estado possibilita aos seus cidadãos fazerem aquilo que ele não pode fazer por conta própria, ou seja, confiarem uns nos outros. 
Os princípios de volume e intensidade do capital social fundem-se nos demais insumos da vida social, proporcionando um fluxo multilateral nas mais diversas estruturas, influenciando ações e resultados, seja ele na esfera individual ou coletiva. Tais processos de confiança mútua dentro de redes de relações interpessoais tornaram-se primordiais após a segunda guerra mundial e o imediato objetivo de reconstrução global. Nesse período concentram-se esforços para alavancar o desenvolvimento mundial tendo como suporte agências internacionais, tais quais a Organizações das Nações Unidas (ONU), UNESCO, Organização Mundial da Saúde (OMS), Fundo das Nações Unidas para a Infância (UNICEF), Fundo Monetário Internacional (FMI) e Banco Internacional para Reconstrução e Desenvolvimento (BIRD), que mesmo após o período do pós-guerra tornaram-se os principais divulgadores e fomentadores de projetos e programas cujo foco fosse o avanço econômico atrelado ao aparato das estruturas sociais com ênfase no capital humano e capital social, como ocorre ainda hoje.

De acordo com D’Araújo (2010), o Banco Mundial a partir dos anos de 1990 adotou uma série de variáveis a serem levadas em consideração na avaliação de seus financiamentos para projetos de desenvolvimento social. Segundo a autora, o banco focaliza em sua análise quatro modos de capital: Capital Natural (recursos naturais do país); Capital Financeiro (infraestrutura, bens de capital imobiliário e financeiro); Capital Humano (educação, saúde e bem-estar da população); e Capital Social (laços de cooperação e confiança social). Esse modelo definido pelo Banco Mundial tende a dar ênfase e fôlego para ações descentralizadas cujo cerne concentra-se na própria sociedade civil. Esse modelo, altamente apoiado pelos organismos internacionais, tem como parâmetro o estudo longitudinal de Putnam (2006) e as correlações positivas encontradas entre o capital social e o desenvolvimento local.

Para Putnam (2006), a manutenção do status quo de desenvolvimento diferenciado entre regiões da Itália mesmo após uma grande mudança de concepção política e participação cívica, deveu-se pelo que ele chamou de capital social, e o forte elo de confiança e reciprocidade civil muito mais presente na região norte do que na região sul. De acordo com o autor:

A superação dos dilemas da ação coletiva e do oportunismo contraproducente daí resultante depende do contexto social mais amplo em que determinado jogo é disputado. A cooperação voluntária é mais fácil numa comunidade que tenha herdado um bom estoque de capital social sob a forma de regras de reciprocidade e sistemas de participação cívica. Aqui o capital social diz respeito a características da organização social, como confiança, normas e sistemas, que contribuam para aumentar a eficiência da sociedade, facilitando as ações coordenadas. (PUTNAM, 2006, p.177)

Esse desenvolvimento mais acentuado da região norte em relação à região sul da Itália apresentou importantes indicativos de reflexão sobre o capital social, que pode direcionar ações coletivas em prol do desenvolvimento regional tendo como parâmetro não apenas elementos econômicos, mas também elementos da vida cotidiana como educação, saúde e trabalho.

Em outra perspectiva, Fukuyama (1996) acredita que a "confiança" é o fator primordial do capital social como base para o desenvolvimento. Para ele a confiança garante uma aproximação entre as ações da sociedade gerando um ciclo de prosperidade quando associado aos fatores sociais locais e aspirações individuais. Dessa forma as ações e decisões devem, por conseguinte, embasar-se no atual momento da sociedade levando em consideração seu status e possível feedback frente a imposições que possam, diretamente, afetar o capital social imediato. Nessa perspectiva, Fukuyama (2002) afirma que o capital social atua dentro do processo de desenvolvimento, como um suporte crítico à sociedade. 
A construção do elemento confiança dentro de uma sociedade aparece, portanto, como cadeias de relações sociais que se multiplicam com o uso e tendem a se extinguir com o desuso, dessa forma, o capital social constitui-se na sociedade na medida em que os laços de confiança são fortalecidos entre os indivíduos e que as relações sociais tornam-se mais amplas e frequentes, tendo o que Albert Hirschman² denominou de "recursos morais". Quanto mais duas pessoas confiam uma na outra, maior a confiança mútua; no entanto, quanto maior a desconfiança maior serão as atitudes que valorizam a própria desconfiança. (PUTNAM, 2006, p.179)

\section{Estudos em foco}

O presente estudo caracteriza-se por utilizar a abordagem qualitativa (THOMAS, NELSON e SILVERMAN, 2012), tendo como parâmetro de investigação o uso da revisão sistemática de literatura. A revisão sistemática, assim como outros tipos de estudo de revisão, é uma forma de pesquisa que utiliza como fonte de dados a literatura sobre determinado tema (SAMPAIO, MANCINI, 2006).

Dessa forma definiu-se como pergunta norteadora para o processo inicial de busca a seguinte indagação: as estruturas que fundamentam o capital social poderiam influenciar em ações no campo da atividade física com vistas à saúde?

Esse questionamento, a partir dos pressupostos teóricos sobre a definição do conceito de capital social, norteou a busca por referenciais que trouxessem uma luz sobre a possível direta relação estabelecida com a prática de atividade física com vistas à saúde. Assim foi definida que a base de dados utilizada para a busca seria a da Elsevier (ScienceDirect), sendo o primeiro passo marcado pela estratificação a partir das informações constantes nos títulos, descritores e abstract, a partir do método booleano de operação lógica. Os descritores "social capital", "physical activity" e "health" foram associados aos operadores lógicos "and", "+", e os resultados obtidos podem ser observados na tabela 1.

Tabela 1 - Resultado da busca por artigos

\begin{tabular}{|l|l|l|}
\hline \multicolumn{1}{|c|}{ Descritor } & Operador Booleano & \multicolumn{1}{c|}{ Resultado } \\
\hline Social Capital, Health & $+/$ and & 760 \\
\hline Social Capital, Physical Activity & $+/$ and & 78 \\
\hline Social Capital, Physical Activity, Health & $+/$ and & 8 \\
\hline
\end{tabular}

A partir dos resultados postos, definiu-se a utilização dos estudos que abordavam todas as perspectivas de associação entre capital social, atividade física e saúde, reduzindo o escopo para apenas oito artigos. Dessa forma, partiu-se para a leitura dos resumos das referidas publicações para comprovar a perspectiva de associação entre os temas, verificando também o desenho do estudo, o tamanho da amostra e a significância observada ou relevância declarada. Em um primeiro momento notou-se que existe ainda uma lacuna estabelecida na literatura com referência ao estudo da teoria do capital social e suas implicações no campo da atividade física. Grande parte das publicações disponibilizadas na base de dados ScienceDirect concentra-se em poucos países, sendo o primeiro estudo encontrado datado de 1999.

\footnotetext{
2 1915-2012 - Economista estadunidense de origem alemã cujas principais obras versaram sobre política econômica e ideologia política. Para maiores informações sobre a biografia e obras de Hirschman ver: LEPENIES, Philipp H.. Possibilismo: vida e obra de Albert O. Hirschman. Novos estudos - CEBRAP, São Paulo, n. 83, Mar. 2009. Disponível em: < http://dx.doi.org/10.1590/S0101-33002009000100005>. Acesso em: 21 abr. 2015
} 
O segundo passo foi realizar a leitura em sua totalidade, gerando importantes pontos de reflexão sobre a relação entre o capital social e o possível impacto no comportamento relacionado à atividade física com vistas à saúde. A última etapa concentrou-se em relacionar os resultados obtidos nos estudos selecionados na base de dados ScienceDirect com os pressupostos centrais da teoria do capital social, verificando a sua adequação central e aderência ao tema. Sobre esse aspecto, cabe ressaltar que o princípio defendido por Putnam (2006) indica o principal ponto possível de convergência entre os temas.

De acordo com o proposto por Putnam (2006), fatores associados ao capital social definem conjuntos de recursos que sustentam o nosso próprio bem-estar e o da comunidade ao qual pertencemos. O capital social no seio de uma comunidade pode, de acordo com o autor, estar figurado nas normas aceitas coletivamente, na inclusão social, nas prósperas relações interpessoais, na participação cívica, na igualdade de gênero, na coesão social, na segurança e no suporte à compreensão e à paz. Tais estruturas embasariam o grau de interação entre os entes em determinada comunidade que dessa forma levariam a processos de desenvolvimento autônomos que potencializariam a adesão coletiva a condutas positivas, dentre elas a prática da atividade física.

A tabela 2 apresenta a perspectiva de cada pesquisa a partir de seus resultados, bem como o tamanho no $\mathrm{N}$ amostral e o tipo de desenho metodológico empregado.

Tabela 2 - Caracterização das pesquisas incluídas no estudo

\begin{tabular}{|c|c|c|c|}
\hline Autores & Perspectiva & N Amostral & Desenho \\
\hline $\begin{array}{l}\text { KAWACHI, } \\
\text { KENNEDY, } \\
\text { GLASS (1999) }\end{array}$ & $\begin{array}{l}\text { Existe forte correlação entre } \\
\text { capital social e estado de saúde }\end{array}$ & 167.259 sujeitos & Survey \\
\hline $\begin{array}{l}\text { LINDSTROM, } \\
\text { HANSON, } \\
\text { OSTERGREN } \\
(2001)\end{array}$ & $\begin{array}{l}\text { A condição } \\
\text { bem como o capioeconômica, } \\
\text { acumulado, social } \\
\text { determinantes para a prática de } \\
\text { atividade física. }\end{array}$ & 28.098 sujeitos & Survey \\
\hline $\begin{array}{l}\text { LINDSTROM, } \\
\text { MOGHADDASSI, } \\
\text { MERLO (2003) }\end{array}$ & $\begin{array}{l}\text { O entorno social é determinante } \\
\text { para inatividade física das } \\
\text { pessoas, fato que poderia ser } \\
\text { reduzido com aumento do } \\
\text { capital social local. }\end{array}$ & 5.542 sujeitos & Survey \\
\hline $\begin{array}{l}\text { FERLANDER } \\
(2007)\end{array}$ & $\begin{array}{l}\text { Redes de relacionamentos } \\
\text { fortes tendem a afetar } \\
\text { condições psicológicas de } \\
\text { saúde e seus mecanismos. }\end{array}$ & --- & Teórico \\
\hline $\begin{array}{l}\text { SAPAG, } \\
\text { KAWACHI (2007) }\end{array}$ & $\begin{array}{l}\text { O capital social potencializa a } \\
\text { promoção e saúde, favorecendo } \\
\text { o empoderamento das } \\
\text { comunidades sobre a temática. }\end{array}$ & --- & Bibliográfico \\
\hline $\begin{array}{l}\text { FUJIWARA, } \\
\text { KAWACHI (2008) }\end{array}$ & $\begin{array}{l}\text { A confiança, sentimento de } \\
\text { pertença e participação } \\
\text { comunitária } \\
\text { associação significativa com o } \\
\text { estado de saúde. }\end{array}$ & 1.888 sujeitos & Survey \\
\hline UESHIMA et al. & $\begin{array}{l}\text { Aumento da promoção da } \\
\text { prática de atividade física por }\end{array}$ & 4 mil sujeitos & Survey \\
\hline
\end{tabular}




\begin{tabular}{l|l|l|l}
\hline (2010) & $\begin{array}{l}\text { meio do incremento de capital } \\
\text { social das comunidades. }\end{array}$ & & \\
\hline RAMLAGAN, & $\begin{array}{l}\text { Fatores relacionados ao capital } \\
\text { social possuem associação com } \\
\text { PELTZER, }\end{array}$ & \multirow{2}{*}{3.840 sujeitos } & Survey \\
PHASWANA- & $\begin{array}{l}\text { meres condições de saúde } \\
\text { dos sujeitos. }\end{array}$ & & \\
\hline
\end{tabular}

Observou-se que os desenhos metodológicos, em sua grande parte, realizados através de surveys, indicam estudos de caráter descritivos, e apenas dois casos com estudos de natureza teórica conceitual. Núcleos e grupos de pesquisa do Japão e da Suécia concentram grande parte da produção temática da área.

\section{Pontos da análise encontrados na relação entre Capital Social e Atividade Física}

O conceito de capital social está associado à forma como a sociedade, estratificada em pequenos grupos caracterizados como comunidades a partir de seus próprios perfis, ampliam ou retrocedem em relação a intensidade de suas conexões internas, bem como a qualidade e quantidade dessas interações. Dessa forma, compreender o significado do termo comunidade dentro de uma sociedade nessa perspectiva é o principal ponto de partida para analisar, a partir de então, as relações dela provenientes.

Para Chau (2007), o termo comunidade faz referência à: 1) sentimento de identidade e pertença, tipificado por adesão de sujeitos a um grupo; 2) composição de símbolos comuns (linguagem e práticas sociais); 3) compartilhamento de normas e valores; 4) sentimento de reciprocidade coletiva ou mútua influência; 5) compromisso de atender necessidades compartilhadas entre os entes, e 6) compartilhamento emocional.

Assim, a transmissão de conhecimentos compartilhados socialmente aliado à participação cívica nas comunidades e o compromisso social estabelecido a partir da confiança gerada entre os entes poderiam facilitar uma possível redução dos níveis de inatividade física. Ter uma rede de relacionamentos (amigos, parentes, vizinhos) que são favoráveis à prática de atividade física potencializaria ações conjuntas de valorização comunitária, favorecendo e fortalecendo esse campo de atuação para o desenvolvimento individual e coletivo. Nessa perspectiva, existe o entendimento de que as relações sociais proporcionadas e fomentadas em uma determinada comunidade podem, a partir do grau de confiança e reciprocidade geradas a partir das mesmas, assegurar níveis reduzidos de inatividade física mesmo quando variáveis como: sexo, idade, estrutura familiar, autopercepção de saúde, e estado mental são controladas. (UESHIMA et al., 2010)

De acordo com Lindstrom, Moghadassi e Merlo (2003), o baixo índice de participação em programas de atividade física, bem como a direta relação estabelecida com a inatividade física de comunidades inteiras está associado à baixa renda, baixo nível de escolaridade e baixo nível socioeconômico. Essa perspectiva confirma o pressuposto de que o acesso à educação e melhores condições socioeconômicas resultariam em um capital social elevado, favorecendo uma rede estruturada de relacionamentos comunitários que fortaleceria ações de cunho individual e coletivo, atuando de forma a retroalimentar o próprio sistema. Dessa forma, a ausência desse mesmo fator impactaria negativamente de forma proporcional a baixa adesão a ações que facilitem o desenvolvimento social e individual. Os baixos níveis socioeconômicos, de escolaridade e de renda, refletiriam, portanto, em um capital social fragmentado, fato que, de acordo com Lindstrom, Hanson e Ostergrem (2001), tornam-se cada vez mais importantes como fatores explicativos para as diferenças em relação ao estado de saúde. 
Lindstrom, Hanson e Ostergrem (2001) sugerem ainda de forma direta a influência do capital social na ligação entre status socioeconômico e comportamento relacionado à saúde. Altos níveis de capital social poderiam, assim, aumentar a capacidade do indivíduo para influenciar fatores determinantes e relevantes para o futuro de sua saúde. É plausível que o acesso a um elevado nível de capital social, o que implica um forte sentido de ser capaz de influenciar a própria saúde, portanto, aumenta o grau de adesão à atividade física.

Essa posição é corroborada com o estudo anterior de Kawachi, Kennedy e Glass (1999), que apontam amplas evidências de que indivíduos isolados, que não estão inseridos em uma comunidade, estão mais suscetíveis a terem maus resultados de saúde por causa de acesso limitado a recursos, apoio moral e ajuda direta. Tais resultados seriam em decorrência do esgotamento do capital social local em ambientes onde as oportunidades de gerar laços sociais são limitadas.

Na mesma linha de raciocínio, Fujiwara e Kawachi (2008), apontam para estudos que convergem no sentido de assegurar que o grau de capital social de determinada comunidade está diretamente relacionado com resultados de saúde física, saúde mental e comportamento. No entanto, os autores destacam a necessidade de compreensão profunda das características sociais em análise, uma vez que as percepções sobre saúde, fatores genéticos e traços de personalidades podem impactar no entendimento sobre a intensidade do capital social local.

A forma como comunidades atuam frente à valoração de ações conjuntas de reciprocidade, pertencimento e confiança acabam por direcionar pautas de interesse coletivo em diferentes áreas, dentre as quais a própria atividade física a partir da perspectiva da saúde. De acordo com Ferlander (2007), o elevado nível de capital social de determinada comunidade poderia influenciar questões políticas no campo da saúde, inferindo ações de cunho diversas, gerando mecanismos potencializadores no campo da atividade física por meio do incentivo institucional. Essa possibilidade pautada em valoração das conexões comunitárias é posta por Sapag e Kawachi (2007) como um processo de empoderamento onde o capital social é ao mesmo tempo recurso e objetivo, sendo impossível desassociá-lo das estruturas políticas e sociais que permeiam as ações dos indivíduos na sociedade.

Mas afinal, como classificar o capital social nesse contexto de interações e objetivos, tanto individuais quanto coletivos? Fukuyama (2002) considera o capital social como qualquer tipo de instância onde as pessoas cooperam para objetivos comuns com base em normas e valores informais compartilhados. Como um dos resultados do somatório de tais premissas, a confiança interpessoal, classificada por Putnam (2006) como pré-requisito para a participação política, obediências às leis e de tolerância às diversidades, emerge como um indicador que dá ao escopo do capital social um ciclo virtuoso que, de acordo com Rennó (2001), uma vez estabelecido, tende a continuar no tempo, ainda que exija constante exercício.

Sobre tal aspecto destaca-se também o posicionamento de Lindstrom, Hanson e Ostergren (2001) sobre a participação social em práticas de atividade física e lazer em determinadas comunidades. Para os autores, as questões relativas ao acúmulo de capital social local e à direta participação em ações voltadas à atividade física devem ser consideradas a partir de barreiras "internas" e "externas" que se modificam em diferentes grupos socioeconômicos. As barreiras internas fariam referência a questões de motivação e falta de tempo (mais propensa a grupos socioeconômicos mais elevados), enquanto que barreiras externas fariam referência à falta de recursos financeiros, ausência de transporte e doença (mais propensa a grupos socioeconômicos mais baixos).

Assim, a estrutura que sustenta o capital social de determinada comunidade, deve, portanto, ser analisada pelo prisma das conexões estabelecidas entre interesse coletivo e necessidades individuais. Dessa forma, o clico propiciado por iniciativas comunitárias de 
prática de atividade física sugere que o apoio coletivo seria eficaz para aumentar a quantidade e o tempo gasto envolvido com tal prática, na mesma medida em que a tipificação da rede de relacionamentos, com suas características e graus de interações e frequências, qualificariam o engajamento social à causa (CHAU, 2007). Pondera-se, a partir de tais pressupostos, que a efetiva adesão a tais iniciativas só é concretizada de forma contundente a partir da livre escolha individual, que, no entanto, como nos afirma Lindstrom, Hanson e Ostergren (2001) e Putnam (2006), pode ser resultado de pressão ou incentivo de atores com elevada participação social.

Para além das especificidades locais que podem resultar na direta participação individual no engajamento cívico, o entendimento de que a participação social na tomada de decisões da vida coletiva pode ser um importante passo para a mudança de comportamento frente a determinadas circunstâncias, e pode corroborar para a mudança de parâmetros entre indivíduos. Conforme aponta D’Araújo (2010):

A participação comunitária/cívica pode envolver uma grande gama de atividades: atuação em vários tipos de associações comunitárias, clubes de esporte, lazer e arte, partidos políticos, sindicatos, cooperativas, entre outros. Estas participações tendem a aumentar as possibilidades de cooperação horizontal entre os membros, já que o grau de pertencimento pode promover regras de reciprocidade, redimensionando a confiança e possibilitando colaborações entre os participantes. (D'ARAÚJO, 2010, p.19)

O nível de interação entre os indivíduos a partir de um objetivo em comum pode potencializar reflexões sobre estratégias que permitam, por parte das comunidades, a adoção de um melhor nível de saúde a partir de hábitos próprios. Essa questão é posta pela própria OMS (2013, apud RIBEIRO, 2015), que entende que todas e quaisquer combinações de experiências de aprendizagem concebidas para ajudar os indivíduos e as comunidades a melhorar a sua saúde, através do aumento do seu conhecimento, ou, influenciando as suas atitudes, deve ser valorizada tomando como referência um princípio de educação para a saúde. Tal posição vai de encontro ao estudo de Ramlagan, Peltzer e Phaswana-Mafuya (2013) que encontrou relações significativas entre estado de saúde e capital social em idosos na África do Sul.

O estudo de Ueshima et al. (2010) apontou para o fato de que a participação social pode ter um efeito protetor frente à inatividade física de indivíduos, fato que para os autores coloca o capital social, e especialmente a questão da confiança, como o divisor de águas em relação aos princípios de adesão e engajamento a ações autônomas e/ou orientadas de prática de atividade física com vistas à melhoria do estado de saúde.

Com o aumento do capital social de comunidades a partir da evolução das relações com vistas à prática regular de atividade física, entende-se que o ciclo virtuoso de manutenção de tais estruturas vigentes, defendido por Rennó (2001), subsidiará o surgimento de novos desafios comunitários de participação democrática, o que poderá influenciar novas ações no campo da própria atividade física.

\section{Considerações Finais}

O capital social, fator resultante dos objetivos, princípios, intensidades e qualidades das relações sociais estabelecidas entre pessoas de uma determinada comunidade, configuram-se como um indicador que pode potencializar a participação popular em ações voltadas para a prática da atividade física como fator de mudança de comportamento.

Essa possibilidade tenderia a ser cada vez mais forte a partir do momento em que a compreensão das comunidades sobre os benefícios da prática de atividade física tornar-se-iam 
presentes na realidade dos indivíduos. No entanto, destaca-se o posicionamento de Putnam (2006), que faz o alerta sobre a manutenção do capital social. Para o autor, as relações sociais internas a uma comunidade possuem a tendência de, ou se ampliar e se solidificar quanto mais os indivíduos confiam e acreditam uns nos outros, ou, reduzir-se e fragmentar-se quanto mais os indivíduos não compartilham experiências e não confiam uns nos outros, gerando o que podemos denominar de capital social negativo na área.

Tomando como referência esse contexto, observamos que dentre os três fatores de apropriação final do capital social pela sociedade definidos por Norma Nie, Jane Junn, e Kenneth Stehlik-Bany (1996), o fator cumulativo resultante é o que traria mais benefícios diretos associados à redução de inatividade física, porém, tal mecanismo só seria eficiente a partir do momento em que ações descentralizadas com foco em estímulos e participações coletivas fossem colocadas em prática por meio dos interesses sociais imediatamente identificados em cada comunidade. Como exemplo de tais mecanismos, pode-se considerar ações de orientação direta em locais propensos à prática de atividade física, bem como a inserção de profissionais da área em equipes multidisciplinares de atenção à saúde em determinadas comunidades.

Assim, considera-se que a atividade física tende a promover de maneira direta nas comunidades a cooperação e a reciprocidade, o aumento das conexões sociais, a identidade coletiva e a confiança interna, fatores esses caracterizados como os pilares do capital social (UESHIMA et al., 2010). No entanto, o grau de associação a esses fatores e a apropriação deles pelas comunidades está relacionado ao nível de engajamento social aos quais tais grupos de indivíduos estão comprometidos conforme assevera Putnam (2006).

Os estudos analisados trouxeram importantes contribuições para o estabelecimento de um eixo norteador que funde os princípios de reciprocidade, confiança e participação cívica a partir da perspectiva da própria atividade física orientada para fins de melhoria do estado de saúde. Tais indicativos sugerem que o capital social, em maior ou menor quantidade em determinada comunidade, pode impactar diretamente nos resultados de adesão à prática de atividade física e ao estado de saúde autorreferido.

Considero o presente tema propenso a novos estudos tendo em vista a impossibilidade de esgotar o debate apenas nesse esboço. A participação de outros pesquisadores adentrando na temática e contribuindo com proposições teóricas sobre os modelos de relações estabelecidas entre capital social e atividade física no Brasil é fundamental para o aperfeiçoamento da área.

\title{
INTRODUCTORY NOTES ON THE RELATIONSHIP BETWEEN THE THEORY OF SOCIAL CAPITAL AND PHYSICAL ACTIVITY
}

\begin{abstract}
The objective of this review was to try to establish possible associations between the theory of social capital and physical activity from international studies published in ScienceDirect database. Based on the qualitative approach, and using the systematic review process of literature, we sought through logical descriptors and operators papers with the proposed theme. It analyzed the social capital from the structure and intensity that underpins the social relations of a community from the trust, solidarity, reciprocity and civic participation. It is suggested that physical activity and health status may be considered factors resulting from capital accumulation, increasing the creation and maintenance of virtuous circles of popular participation reducing levels of physical inactivity.
\end{abstract}

Keywords: Social Capital; Physical Activity; Health; Community 


\section{NOTAS INTRODUCTORIAS SOBRE LA RELACIÓN ENTRE LA TEORÍA DEL CAPITAL SOCIAL Y LA ACTIVIDAD FÍSICA}

\section{Resumen}

El objetivo de esta revisión fue intentar establecer posibles asociaciones entre la teoría del capital social y la actividad física a partir de estudios internacionales publicados en la base de datos ScienceDirect. Basado en el enfoque cualitativo, y con el proceso de revisión sistemática de la literatura, se buscó a través de descriptores y operadores lógicos artículos con el tema propuesto. Se analizó el capital social a partir de la estructura e intensidad que sustenta las relaciones sociales de una comunidad como la confianza, la solidaridad, la reciprocidad y la participación cívica. Se sugiere que la actividad física y el estado de salud pueden ser considerados factores resultantes de la acumulación de capital social, mejorando la creación y el mantenimiento de círculos virtuosos de la participación popular, reduciendo las tasas de inactividad física.

Palabras Clave: Capital Social; Actividad Física; Salud; Comunidad.

\section{Referências}

BASTOS, Francisco Avelar; SANTOS, Everton; TOVO, Maximiano Ferreira. Capital Social e Sistema Único de Saúde (SUS) no Brasil. Saúde Soc. São Paulo, v.18, n.2, p.177-188, 2009.

CHAU, Josephine. Physical Activity and Building Stronger Communities. Centre of Physical Activities and Health - Cpah, 2007, 19p.

COLEMAN, James Samuel. Social Capital in the Creation of Human Capital. The American Journal of Sociology, vol. 94, Supplement: Organizations and Institutions: Sociological and Economic Approaches to the Analysis of Social Structure. 1988, pp. 95-120.

D’ARAUJO, Maria Celina. Capital Social. 2. ed. Rio de Janeiro, Zahar, 2010, 66p.

FERLANDER, Sara. The Importance of Different Forms of Social Capital for Health. Acta Sociologica. vol.50, june 2007. pp.115-128. DOI: 10.1177/0001699307077654.

FUKUYAMA, Francis. Social Capital and Development: The coming agenda. SAIS Review, volume 22, number 1, Winter-Spring, 2002, pp. 23-37.

FUJIWARA, Takeo. KAWACHI, Ichiro. Social Capital and Health. A Study of Adult Twins in the U.S. American Journal of Preventive Medicine. vol.35, n. 2, 2008, p. 139-144.

HELLIWELL, John; PUTNAM, Robert David. Education and Social Capital. National Bureau of Economic Research. maio 1999, p.1-25. Disponível em:< http://www.nber.org/papers/w7121.pdf?new_window=1>. Acesso em: 13 maio 2015.

KAWACHI, Ichiro; KENNEDY, Bruce; GLASS, Roberta. Social Capital and Self-Rated Health: A Contextual Analysis. American Journal of Public Health, v.89, n.8, ago. 1999, p. 1187-1193. 
LINDSTROM, L; MOGHADDASSI, M; MERLO, J. Social capital and leisure time physical activity: a population based multilevel analysis in Malmö, Sweden. Journal Epidemiol Community Health, 2003, p.57:23-28.

LINDSTROM, Martin; HANSON, Bertil S.; OESTERGREN, Per-Ol of. Socioeconomic differences in leisure-time physical activity: the role of social participation and social capital in shaping health related behaviour. Social Science and Medicine. n. 52. 2001, p. 441-451.

LOCH, Mathias Roberto; SOUZA, Regina Kazue Tanno de. Capital Social: dados descritivos de estudo de base populacional e associação com comportamentos simultâneos de risco à saúde. Revista Brasileira de Atividade Física e Saúde. Pelotas, RS. 19 (6), nov. 2014, pp774-784.

NIE, Norma; JUN, Jane; STEHLIK-BANY, Kenneth. Education and Democratic Citzenship in America. Chicago. University of Chicago. 1996.

ORGANIZAÇÃO MUNDIAL DA SAÚDE. Atividade Física. Folha Informativa. No 385 , Fevereiro. 2014.2 Disponível em:< http://actbr.org.br/uploads/conteudo/957_FactSheetAtividadeFisicaOMS2014_port_REV1.pd f>. Acesso em: 21 abr.2015.

PORTES, Alejandro. Capital social: origens e aplicações na sociologia contemporânea. Sociologia, Problemas e Práticas, Oeiras, n. 33, set. 2000. Disponível em:< http://www.scielo.gpeari.mctes.pt/scielo.php?script=sci_arttext\&pid=S087365292000000200007\&lng=pt\&nrm=iso>. Acesso em: 13 maio 2015.

PUTNAM, Robert David. Comunidade e Democracia: a experiência da Itália moderna. 5. ed. Rio de Janeiro, Editora FGV, 2006, 260p.

RAMLAGAN, Shandir; PELTZER, Karl; PHASWANA-MAFUYA, Nancy. Social capital and health among older adults in South Africa. BMC Geriatrics. 13:100, 2013, pp. 1-11.

RENNÓ, Lúcio R. Confiança Interpessoal e Comportamento Político: microfundamentos da teoria do capital social na América Latina. Opinião Pública, Campinas, v. 7, n. 1, 2001, p.3359.

RIBEIRO, José Luiz Pais. Educação para a saúde. Psicologia, Saúde \& Doenças. Sociedade Portuguesa de Psicologia da Saúde, jan. 2015. DOI: <http://dx.doi.org/10.15309/15psd160102>.

SAMPAIO, R.F; MANCINI, M.C. Estudos de Revisão Sistemática: um guia para síntese criteriosa da evidência científica. Revista Brasileira de Fisioterapia. São Carlos, v.11, n.1, p. 83-97, jan./fev. 2007.

SAPAG, Jaime; KAWACHI, Ichiro. Capital social y promoción de la salud en América Latina. Revista de Saúde Pública. 2007; 41(1):139-149.

THOMAS, JERRY R; NELSON, JACK K; SILVERMAN, STEPHEN J. Métodos de Pesquisa em Atividade Física. 6. ed. Porto Alegre. Artmed, 2012, 478p. 
UESHIMA, Kazumune; et al. Does Social Capital Promote Physical Activity? A PopulationBased Study in Japan. Plos One, ago. 2010, v. 5, issue 8, pp.1-6.

UNESCO. Diretrizes de la UNESCO para el reconocimiento, validación y acreditación de los resultados del aprendizaje no formal e informal. Instituto de la Unesco para el Aprendizaje a lo Largo de Toda la vida. Alemanha, 2012, 10 p.

Recebido em: 28/07/2015

Revisado em: 23/11/2015

Aprovado em: 01/12/2015

Endereço para correspondência:

bendrath@gmail.com

Eduard Angelo Bendrath

Universidade Estadual de Maringá

Departamento de Educação Fìsica.

Praça Independência

Centro

86870-000 - Ivaiporã, PR - Brasil 